\title{
Letter from the Editors: AI Magazine in the new era of AI
}

It is a great honor for us to take the baton from Ashok Goel in becoming editors-in-chief of this magazine. For one of us (K. Brent Venable), the involvement with AI Magazine dates to 2014 when she joined the editorial board as a reviewer first and then in 2018 as an associate editor. The other (Odd Erik Gundersen) started as a columnist writing the column on reproducibility in 2018 before becoming an associate editor in 2020 and finally the co-editor in chief with Brent in 2021.

Both of us have witnessed the tremendous dedication and work with which David first and then Ashok have navigated AI Magazine during times of fundamental change. We pick up the work at a very strong position in particular thanks to the successful re-shaping of AI Magazine that Ashok has accomplished in recent years. The ambitious agenda he tirelessly carried out has made AI magazine a leading source of information and news on AI and its sub-disciplines, and a forum for debates and discussion on social, cultural and economical aspects of AI. Most importantly it has also started the Interactive version of $A I$ Magazine de facto initiating the transition of the Magazine to a digital platform.

We take this opportunity to thank David Leake and Ashok Goel and their editorial teams for their tremendous work and dedication!

We embark on this new editorial journey with an outstanding editorial board including a fine-tuned balance of legacy and new members. In particular, we thank our associated editors Sandip Sen and Michael Wollowski for the professional and timely handling of general track articles; Robert Morris for his efficient management of ever growing number of columns, Pearl $\mathrm{Pu}$ for her detailed oversight of the special issues and Joshua Eckroth for being the driving force behind Interactive AI Magazine. A particular thanks goes to our absolutely essential editorial assistant, Ida Camacho, who keeps all of us in line and organized with her proactive and skillful approach. Thank you Ida!

We would also like to thank the executive director of AAAI, Carol Hamilton, and Mike Hamilton for all their help in the transition between editors. Mike Hamilton has stepped down as the publishing director after more than 30 years of ensuring that AI Magazine is published on time and looks and feels exactly like $A I$ Magazine. Thank you for all the hard work. We hope you had some fun as well.

This editorial letter is part of an issue of which we are particularly proud of. The main theme is "Recommender Systems": unquestionably one of the scientific success stories which have generated some of the most pervasive technological tools that have brought AI into the life of so many individuals. The issue features a first set of six papers on this subject, covering interesting topics including the role of reinforcement learning in automated recommendations, HCI, the importance and challenges tied to reproducibility in recommender systems and the latest trends in this area. We are deeply grateful to guest editors Dietmar Jannach, Pearl Pu, Francesco Ricci and Markus Zanker for putting together this compelling line-up. This issue also contains an interesting report on the Third international Competition on computational Models of Argumentation by Stefano Bistarelli, Lars Kotthof, Francesco Santini and Carlo Taticchi) as well as two columns addressing the timely topic of Engagement during Pandemic Teaching by Michael Wollowski and Betting on Bets by Chris Welty, Praveen Paritosh and Kurt Bollacker.

$\mathrm{AI}$ is at an all-time-high in terms of success, expectations but also inevitably criticisms. The proliferation of content both legitimately and illegitimately tied to AI makes it a priority to have a venue where the content delivered is scientifically sound. AI Magazine is in a unique position to fulfill this role as it was initially conceived as the voice of the AAAI community, a community that has played a foundational role in the scientific development of AI.

Cognizant of how the role of AI has recently evolved, moving well beyond the realm of academic discussion we identify several priorities of AI Magazine. In 2001 the AAAI conference had 400 submissions, and in 2021 it neared 10 000 submissions. This is just an example that shows how much the AI research community has grown. The evolution of AI's role in society is maybe best reflected by the fact that more than thirty nations have written their own national AI strategies over the past couple of years. Several international strategies have been made as well, most notably from the UN, EU and the G7 nations. All continents are represented with the exception of Antarctica. 
As our first goal, we set out to make AI Magazine a venue that better reflects the current state of AI. AI Magazine needs to represent the wider geographical scope and international breadth of the AI community. AAAI has spearheaded several initiatives to promote diversity and inclusion in AI. Indeed, AAAI 2021 had a dedicated track to address this theme. We strongly believe AI Magazine should reflect this priority as well. We plan to pay careful attention to ensuring a diversity of the published content and the editorial team. Thus, we will devote special attention to include contributions from fellow women researchers and researchers belonging to minorities while keeping a balance in nationality and geographical representation as we redesign AI magazine in light of the AI evolution.

AI Magazine has uniquely positioned itself as an ideal venue where senior researchers can more freely debate and discuss controversial topics as well as global trends of the discipline. We plan to continue this tradition aided in particular by our world class advisory board which includes numerous AAAI and EurAI fellows as well as several leaders in the field from industry and academia. We want to make AI Magazine a place where personal views on AI-related issues involving societal, political, ethical and economical considerations can find an outlet after undergoing a thorough review in terms of scientific soundness.

We also plan to devote considerable effort in making AI Magazine appealing to a younger crowd by further enhancing our more modern and nimble forms of publishing via Interactive $A I$ Magazine. As a sneak peek on the many innovations the editorial board has planned, we are delighted that, starting Spring 2022 our readers will be able to enjoy AI magazine exclusively in digital form. In addition the role of Interactive AI Magazine will be greatly expanded to include, for example, videos related to articles appearing on AI magazine and "ask me anything"sessions with leaders in the field. We are always seeking new columns on timely and important topics, so please do not hesitate to approach us if you have an idea.

The discipline of AI, the AAAI community and AI Magazine are all undergoing significant and fast paced changes. We are both anxious and excited about steering AI Maga- zine through the transition to a new publishing platform and novel forms of content delivery.

However, the success of AI Magazine has been and always will be determined by you: our incredible community of contributors and readers! We want to take this opportunity to thank all of you! We are also of course eager to receive suggestions, feedback and ideas for improvement at bvenable@ihmc.org and odderik@ntnu.no.

K. Brent Venable ${ }^{1}$ Odd Erik Gundersen ${ }^{2}$

${ }^{1}$ Florida Institute for Human and Machine Cognition, Pensacola, Florida

${ }^{2}$ Norwegian University of Science and Technology Trondheim, Norway

Email: bvenable@ihmc.org, odderik@ntnu.no

\section{AUTHOR BIOGRAPHIES}

$\mathbf{K}$. Brent Venable is a computer scientist with expertise in artificial intelligence. Her research is dedicated to providing a solid framework for the design and deployment of intelligent systems able to reason about preferences. As preferences are fundamental for the analysis of human choice behavior, they are becoming of increasing importance for computational fields such as artificial intelligence (AI), databases, and humancomputer interaction. Dr. Venable joined IHMC in August 2012. Since 2019 she is the director of the newly established IHMC-UWF PhD program in Intelligent Systems and Robotics. She is also professor of computer science at University of West Florida.

Odd Erik Gundersen is an adjunct associate professor at the Norwegian University of Science and Technology and the Chief AI Officer at TrønderEnergi, a Norwegian renewable energy company. Gundersen has applied AI in industry, mostly for startups, since 2006. Currently, he is investigating how AI can be applied in the renewable energy sector and for driver training as well as how research in AI can be made reproducible. 